\title{
Perioperative antibiotic prophylaxis and prosthetic valve endocarditis
}

\author{
C. WARD \\ M.D., M.R.C.P.
}

\author{
A. E. JEPHCOTT \\ M.A., M.B., M.R.C.Path., Dip.Bact.
}

\author{
C. A. HaRdisty \\ B.Sc., M.R.C.P. \\ Cardiothoracic Unit and Public Health Laboratory, \\ Northern General Hospital, Sheffield 5
}

\begin{abstract}
Summary
A study of twenty-five cases of prosthetic valve endocarditis suggests that the antibiotics used for perioperative prophylaxis may alter the type and antibiotic sensitivity of organisms which subsequently infect the artificial valves. Based on the results of this study, the authors have been able to modify their prophylactic regime to encompass these organisms and to predict the antibiotics most likely to be effective in the treatment of prosthetic valve endocarditis in their unit. No single prophylactic or therapeutic regime will be equally effective in all centres, but by examining the different types and sensitivities of bacteria which cause prosthetic valve endocarditis in a locality, antibiotic regimes can be chosen which best suit the local situation.
\end{abstract}

\section{Introduction}

Prosthetic valve endocarditis is a major hazard for patients with artificial cardiac valves. It occurs in approximately $4 \%$ of cases and has a high mortality, particularly during the early postoperative months. Perioperative antibiotic prophylaxis probably reduces the incidence of prosthetic valve endocarditis but, while eradicating some organisms, such therapy may encourage the overgrowth of other, insensitive, strains. If this is so, antibiotics used perioperatively may predetermine the types of organisms which subsequently infect the patients' valves. This hypothesis has not been tested because most centres have used a variety of prophylactic regimes (Dismukes et al., 1973; Hairston and Lee, 1970; Madison et al., 1975; Slaughter, Norris and Starr, 1973; Stein, Harken and Dexter, 1966) and this has obscured the long term effects of specific antibiotics. In this report, the characteristics of prosthetic valve endocarditis occurring in the relatively uniform context of only two related regimes of perioperative antibiotic prophylaxis are described and the implications discussed.
Details of antibiotic prophylaxis

The two regimes used were: (1) ampicillin and cloxacillin $500 \mathrm{mg}$ four times daily of each for 10 days commencing $48 \mathrm{hr}$ before operation; (2) cloxacillin $500 \mathrm{mg}$ four times daily for 5 days beginning immediately after operation.

Details of patients studied, time of infection and outcome (Tables 1 and 2)

Prosthetic valve endocarditis occurred in twentyfive $(3.4 \%)$ of 746 patients who underwent valve replacement during 1970-1975. Average age was 47.7 years. Nineteen were male (average age $49 \cdot 1$ years) and six were female (average age $40 \cdot 8$ years). Affected valves were aortic, seventeen cases; mitral, five cases; aortic plus mitral, three cases.

Thirteen $(52 \%)$ patients died-eleven male and two female - average age 50.9 years.

The time interval between valve insertion and diagnosis of endocarditis varied from 1 to 57 months (average $15 \cdot 1$ months). The average time between operation and diagnosis of infective endocarditis in survivors was 19.8 months and in fatal cases was $11 \cdot 3$ months.

Six $(25 \%$ of cases) presented within 2 months of surgery and of these, four died. A total of fifteen cases occurred within 1 year of surgery and ten of these died. Ten cases occurred more than 1 year after surgery, of whom only three died.

\section{Bacteriology (Tables 2 and 3)}

Table 2 records the organisms encountered and how they relate to the time between valve insertion and the development of infective endocarditis.

Table 3 relates antibiotic sensitivity to the time at which endocarditis occurred and to specific organisms. There were no significant differences between the two regimes of prophylaxis with respect to outcome, organisms cultured or antibiotic sensitivity. 
TABLE 1. Details of twenty-five patients with prosthetic valve endocarditis

\begin{tabular}{|c|c|c|c|c|c|c|c|c|c|c|c|c|c|}
\hline & \multirow{2}{*}{$\begin{array}{c}\text { Number } \\
\text { of } \\
\text { cases }\end{array}$} & \multirow{2}{*}{$\begin{array}{c}\text { Average } \\
\text { age } \\
\text { (years) }\end{array}$} & \multirow{2}{*}{\multicolumn{3}{|c|}{ Sex }} & \multicolumn{3}{|c|}{$\begin{array}{c}\text { Prosthetic } \\
\text { valve affected }\end{array}$} & \multicolumn{5}{|c|}{ Bacteriology } \\
\hline & & & & & & A & $\mathbf{M}$ & $\mathbf{A}+\mathbf{M}$ & Diphth. & Staph. & S.V. & $\begin{array}{l}\text { Cult. } \\
\text { neg. }\end{array}$ & Others \\
\hline All cases & 25 & $47 \cdot 7$ & 19 & & 6 & 17 & 5 & 3 & 8 & 6 & 2 & 5 & 4 \\
\hline Non-fatal & 12 & $43 \cdot 7$ & 8 & & 4 & 8 & 2 & 2 & 3 & 3 & 0 & 4 & 2 \\
\hline Fatal & 13 & $50 \cdot 9$ & 11 & & 2 & 9 & 3 & 1 & 5 & 3 & 2 & 1 & 2 \\
\hline
\end{tabular}

A, aortic; M, mitral; Diphth., diphtheroid bacilli; Staph., Staphylococcus sp.; S.V., Streptococcus viridans; Cult. neg., culture negative.

TABLE 2. Infecting organisms in prosthetic valve endocarditis

\begin{tabular}{lcc}
\hline & $\begin{array}{c}\text { Number } \\
\text { encountered } \\
\text { during first } \\
\text { postoperative } \\
\text { year }\end{array}$ & $\begin{array}{c}\text { Number } \\
\text { encountered } \\
>1 \text { year } \\
\text { postoperatively }\end{array}$ \\
\hline Drganism & 8 & 0 \\
Siphtheroids & 4 & 2 \\
Streptococcus viridans & 0 & 2 \\
Pseudomonas aeruginosa & 2 & 0 \\
Klebsiella sp. & 1 & 0 \\
Meningococcus & 0 & 1 \\
Culture negative & 0 & 5 \\
\hline
\end{tabular}

Eight cases $(32 \%$ of total) were caused by diphtheroid bacilli; all occurred within 1 year of surgery. Only one was sensitive to penicillin and two to ampicillin. All were sensitive to erythromycin, fucidin, lincomycin and gentamicin. Six cases $(25 \%$ of total) were caused by staphylococci (five coagulase negative, one coagulase positive) of which four occurred within 1 year of valve insertion. Three were sensitive to methicillin, erythromycin, fucidin and cephalosporins. All were sensitive to gentamicin.

The two cases due to Pseudomonas aeruginosa and the single case due to Klebsiella sp. occurred within 1 year of surgery and were resistant to the penicillins and to cephalosporins, erythromycin, fucidin and lincomycin, but were sensitive to gentamicin.
Thus, most organisms encountered within 1 year of surgery were resistant to all penicillins and the cephalosporins.

Two cases due to Streptococcus viridans occurred more than 1 year after surgery; one was sensitive to penicillin.

A single case due to meningococcus occurred 42 months after surgery and was sensitive to all antibiotics tested except lincomycin. Five cases of culture-negative endocarditis occurred all after the first postoperative year.

All organisms tested against gentamicin were sensitive to it and most were sensitive to erythromycin and fucidin. On a weight for weight basis erythromycin and fucidin were usually the mose active antibiotics; this was particularly so wit respect to the diphtheroid bacilli.

\section{Discussion}

There is no objective evidence that the incidence of prosthetic valve endocarditis is reduced by antibiotic prophylaxis at the time of valve surgery; in fact Goodman et al. (1968) and Dismukes et al. (1973) implied that the antiobitics might, in some respects, be harmful. Nevertheless, most clinicians follow the recommendation of Geraci, Dale and McGoon (1963) and use some form of antibiotic prophylaxis. This action is justified by the results of several uncontrolled studies (Amoury, Bowman and Malm,

TABLE 3. In vitro antibiotic sensitivity of organisms from cases of prosthetic valve endocarditis

\begin{tabular}{|c|c|c|c|c|c|c|c|c|}
\hline & \multirow{2}{*}{$\begin{array}{l}\text { Number of sensitive } \\
\text { organisms cultured in } \\
\text { first post-op. year }\end{array}$} & \multirow{2}{*}{$\begin{array}{l}\text { Number of sensitive } \\
\text { organisms cultured } \\
>1 \text { year post-op. }\end{array}$} & \multicolumn{6}{|c|}{ Sensitivity of specific organisms } \\
\hline & & & Diphth. & Staph. & S.V. & Ps. & $K l . \mathrm{sp}$. & Mening. \\
\hline Penicillin G & $1 / 14$ & $2 / 5$ & $1 / 8$ & $1 / 5$ & $1 / 2$ & $0 / 2$ & $0 / 1$ & $1 / 1$ \\
\hline Methicillin & $2 / 6$ & $3 / 4$ & & $3 / 5$ & $1 / 2$ & $0 / 2$ & $0 / 1$ & $1 / 1$ \\
\hline Ampicillin & $2 / 10$ & $3 / 8$ & $2 / 7$ & $1 / 5$ & $1 / 2$ & $0 / 2$ & $0 / 1$ & $1 / 1$ \\
\hline Cephalosporin & $5 / 13$ & $4 / 5$ & $4 / 7$ & $3 / 5$ & $1 / 2$ & $0 / 2$ & $0 / 1$ & $1 / 1$ \\
\hline Erythromycin & $11 / 14$ & $3 / 5$ & $8 / 8$ & $3 / 5$ & $2 / 2$ & $0 / 2$ & $0 / 1$ & $1 / 1$ \\
\hline Lincomycin & $9 / 12$ & $3 / 5$ & $7 / 7$ & $3 / 4$ & $2 / 2$ & $0 / 2$ & $0 / 1$ & $0 / 1$ \\
\hline Fucidin & $9 / 12$ & $3 / 5$ & $7 / 7$ & $2 / 4$ & $2 / 2$ & $0 / 2$ & $0 / 1$ & $1 / 1$ \\
\hline Gentamycin & $13 / 13$ & $3 / 3$ & $7 / 7$ & $5 / 5$ & $2 / 2$ & $2 / 2$ & $1 / 1$ & $1 / 1$ \\
\hline
\end{tabular}

Diphth., diphtheroid bacilli; Staph., staphylococci; S.V., Streptococcus viridans; Ps., Pseudomonas aeruginosa; Kl. sp., Klebsiella sp., Mening., meningococcus. 
1966; Stein et al., 1966). The latter study suggests that the use of anti-staphylococcal antibiotics for prophylaxis has reduced the incidence of prosthetic valve endocarditis caused by that organism in the early postoperative period, from $3.7 \%$ to $0.2 \%$.

The incidence of prosthetic valve endocarditis in the present patients $(3.4 \%)$ is similar to that of others (Dismukes et al., 1973; Madison et al., 1975; Slaughter et al., 1973). The mortality is somewhat lower. In contrast to previous reports, the authors found: (1) a lower incidence of cases occurring within 2 months of operation; (2) similarly low incidence of staphylococcal endocarditis; (3) total absence of cases due to Candida; (4) high incidence of diphtheroid bacillus infections.

Some of these differences may be explained by a suggestion of Dismukes et al. (1973). They predicted that anti-staphylococcal prophylaxis given at the time of cardiac surgery might affect the course of subsequent prosthetic valve endocarditis by encouraging the growth of Gram-negative organisms and fungi which gain access to the body at or soon after operation.

The authors attribute two characteristics ((a) the high incidence of diphtheroid bacillus infections; (b) the antibiotic sensitivity of the organisms they encountered) of their group of cases to the antibiotic prophylaxis used at the time of surgery. Two facts support the first of these contentions:

(1) Diphtheroid bacilli occur commonly on the skin of patients and members of operating teams and can easily gain access to the patient's bloodstream during operation.

(2) In a report of seven cases of diphtheroid endocarditis (Jackson and Saunders, 1973), six had received, as had the present cases, ampicillin and cloxacillin for perioperative prophylaxis. Their infections, as did those in the authors' patients, revealed themselves within 1 year of valve insertion.

Regarding the second contention, the use of penicillins for perioperative prophylaxis is likely to allow the growth of resistant contaminants. Most organisms cultured in the authors' laboratory, especially those encountered in the first postoperative year, were resistant to the penicillin group of antibiotics and to the cephalosporins. Only one of the fifteen organisms cultured within 1 year of operation was sensitive to penicillin and only three were sensitive to methicillin. This high incidence of resistance to the penicillins, which included methicillin-resistant staphylococci, has not been previously reported. In the authors' experience, the penicillins are of little value in treating prosthetic valve endocarditis and they now electively use gentamicin plus fucidin, replacing gentamicin with erythromycin in the presence of significant renal impairment. However, this choice of treatment will not necessarily be successful if different perioperative antibiotic prophylaxis has been employed, or even if the same prophylaxis has been used in a different part of the country, for similar species of bacteria cultured in different areas do not always show the same sensitivities (Dismukes et al., 1973; Madison et al., 1975). It is clear, therefore, that recommendations to use specific antibiotics to treat prosthetic valve endocarditis (Stein et al., 1966; Gray, 1975) may be misleading and are not justified, for they fail to take these variable factors into account.

Thus, although the treatment of prosthetic valve endocarditis is primarily dependent on the antibiotic sensitivity of organisms cultured from individual patients, each centre should also plan its own treatment policy, based on the evaluation of past local experience with antibiotic prophylaxis and with in vitro sensitivity testing of organisms cultured from previous cases. This approach to treatment is particularly important when, as happens in approximately $33 \%$ of all cases, treatment has to be started 'blind'-either because of the gravity of the patient's condition or because of persistently negative blood cultures. Furthermore, identification of the organisms which commonly 'break through' the prophylactic regime allows this cover to be altered so as to be effective against them. To this end, the effect of including erythromycin in their perioperative antibiotic prophylaxis is currently being studied by the authors.

\section{References}

Amoury, R.A., Bowman, F.O., JR \& Malm, J.R. (1966) Endocarditis associated intracardiac prostheses. Journal of Thoracic and Cardiovascular Surgery, 51, 36.

Dismukes, W.E., KARChmer, A.W., BuCKLEy, M.J., Austen, W.G. \& Swatz, M.N. (1973) Prosthetic valve endocarditis. Circulation, 48, 365.

Geraci, J.E., Dale, A.J. \& McGoon, D.C. (1963) Bacterial endocarditis and endarteritis following cardiac operations. Wisconsin Medical Journal, 62, 302.

Goodman, J.S., Schaffner, W., Collins, H.A., Battersby, E.J. \& KoENIG, M.G. (1968) Infection after cardiovascular surgery: clinical study including examination of antimicrobial prophylaxis. New England Journal of Medicine, 278, 117.

GRAY, I.R. (1975) The choice of antibiotic for treating infective endocarditis. Quarterly Journal of Medicine, 44, 449.

Hairston, P. \& LeE, W.H. (1970) Management of infected prosthetic heart valves. Annals of Thoracic Surgery, 9, 229.

JACKSON, G. \& SAunders, K. (1973) Prosthetic valve diphtheroid endocarditis treated with sodium fucidate and erythromycin. British Heart Journal, 35, 931.

Madison, J., Wang, E., Gobel, F. \& Edwards, J.E. (1975) Prosthetic aortic valvular endocarditis. Circulation, 51, 940.

Slaughter, L., Norris, J.E. \& Starr, A. (1973) Prosthetic valvular endocarditis. Circulation, 47, 1319.

Stein, P.D., Harken, D.E. \& Dexter, L. (1966) The nature and prevention of prosthetic valve endocarditis. American Heart Journal, 71, 393. 